\title{
Hybrid classical-quantum dynamics
}

\author{
Asher Peres and Daniel R. Terno * \\ Department of Physics, Technion - Israel Institute of Technology, 32000 Haifa, Israel
}

\begin{abstract}
A hybrid formalism is proposed for interacting classical and quantum sytems. This formalism is mathematically consistent and reduces to standard classical and quantum mechanics in the case of no interaction. However, in the presence of interaction, the correspondence principle is violated.
\end{abstract}

PACS numbers: 03.65.Bz, 03.65.Sq

${ }^{*}$ Electronic mail: peres@photon.technion.ac.il, terno@physics.technion.ac.il 
Quantum mechanics gives exceedingly accurate predictions for atomic and nuclear systems. Classical mechanics is just as successful for planetary motion. Can there be a theory encompassing both classical and quantum mechanics, so that atoms and planets can be treated in a unified way? Indeed, it was speculated long ago that the gravitational field is classical, even though its material sources are quantized [1]. Niels Bohr always insisted that measuring instruments must be described in classical terms [2]. However, Bohr did not provide a dynamical description of their interaction with quantum systems. A dual (classical and quantum) description of measuring instruments is possible [3], but this is not a truly hybrid formalism as proposed below, because in that dual formalism the interaction is always between subsystems of the same kind.

If we try to write equations of motion that combine classical canonical variables and quantum operators, we find that the classical variables, whose values ought to be ordinary numbers, pick up operator components, which is manifestly unacceptable (we shall later show how to overcome this difficulty). There have been numerous claims and counterclaims as to the possibility of existence a consistent classical-quantum formalism. Some

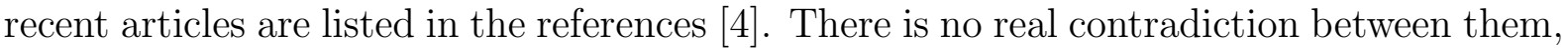
because their conflicting conclusions result from different demands for consistency.

In this article, we propose a hybrid formalism for interacting classical and quantum sytems. This formalism is mathematically consistent and reduces to standard classical and quantum mechanics if there is no interaction between the systems. However, in the presence of interaction, the correspondence principle is violated, so that this hybrid formalism appears physically abnormal.

Our work follows a remark by Jauch [5] that if we assume that not all quantum dynamical variables are actually observable, and if we set rules for distinguishing measurable from nonmeasurable operators, it is then possible to define a classical system as a special type of quantum system for which all measurable operators commute. All the observable dynamical variables of such a classical system can be simultaneously diagonalized and therefore can, in principle, have sharp values. If we are confined to this set of observables and we use a basis where they all are diagonal, then only diagonal elements of density matrices can be reconstructed from measurement statistics and have an operational meaning. Quantum states (i.e., density matrices) with the same diagonal elements are indistinguishable. We may therefore consider all states as pure by defining their off-diagonal elements 
as $\rho_{m n}=\sqrt{\rho_{m m} \rho_{n n}}$, so that $\rho$ always has rank 1. It is then possible to define a classical state vector $\psi$ by $\rho=\psi \psi^{\dagger}$.

Pure states defined in this way do not imply that dynamical variables have sharp values. The situation is just as in classical statistical mechanics: the expectation value of an observable $A$ is given by

$$
\langle A\rangle=\operatorname{tr}(\rho A)
$$

and we may then have $\left.\left\langle A^{2}\right\rangle\right\rangle\langle A\rangle^{2}$, as usual. Note that such a "classical" system also has noncommuting operators, but the latter should be considered as abstract mathematical expressions which are not experimentally observable. With this formal definition, the notion "classical" acquires a meaning with respect to a specified set of dynamical variables. This does not preclude that, by using more elaborate observation means, every physical system might display quantum features.

To completely mimic classical mechanics, we need a quantum algorithm that reproduces exactly the equations of motion of a classical canonical system. For this, we shall follow Koopman's formalism [6, []. We consider for simplicity a single degree of freedom and denote the canonical variables as $x$ and $y$ (rather than $x$ and $p$, as usual, because we wish to reserve the symbol $p$ for the momentum of a quantum system, to be introduced later). Let us write the Liouville equation as

$$
i \partial f / \partial t=L f
$$

where $L$ is the Liouville operator, or Liouvillian,

$$
L=\left(\frac{\partial H}{\partial y}\right)\left(-i \frac{\partial}{\partial x}\right)-\left(\frac{\partial H}{\partial x}\right)\left(-i \frac{\partial}{\partial y}\right) .
$$

The Liouville density $f$ is never negative. Since its quantum analog, namely Wigner's distribution [7, 8] (which may be negative), is quadratic in the quantum wave function, it is convenient to introduce likewise a "classical wave function" $\psi=\sqrt{f}$, which in this case satisfies the same equation of motion as $f$,

$$
i \partial \psi / \partial t=L \psi
$$

We shall now consider $\psi$ as the fundamental entity (but only $f=|\psi|^{2}$ has a direct physical meaning). It can be proved that, under reasonable assumptions about the 
Hamiltonian, the Liouvillian is an essentially self-adjoint operator and generates a unitary evolution [9]:

$$
\langle\psi, \phi\rangle:=\int \psi(x, y, t)^{*} \phi(x, y, t) d x d y=\text { const. }
$$

The introduction of a Hilbert space with unitary dynamics enables one to use familiar methods of quantum mechanics for the analysis of ergodic problems [9] and of classical chaos [10]. It is possible to further mimic quantum theory by introducing commuting operators $\hat{x}$ and $\hat{y}$, defined by

$$
\hat{x} \psi=x \psi(x, y, t) \quad \text { and } \quad \hat{y} \psi=y \psi(x, y, t) .
$$

Note that the momentum $\hat{y}$ is not the shift operator (the latter is $\hat{p}_{x}=-i d / d x$ ). Likewise the boost operator is $\hat{p}_{y}=-i d / d y$. These two operators are not observable. We shall henceforth omit the hats over the classical operators, as there is no danger of confusion.

The analogy with quantum mechanics can be pushed further. What we have above is a "Schrödinger picture" (operators are constant, wave functions evolve in time as $\psi(t)=$ $U(t) \psi(0)$, where $U(t)=e^{-i L t}$ if the Hamiltonian is time-independent). We can also define a "Heisenberg picture" where wave functions are fixed and operators evolve:

$$
X_{H}(t)=U^{\dagger} X U
$$

The Heisenberg equation of motion,

$$
i d X_{H} / d t=\left[X_{H}, L_{H}\right]=U^{\dagger}[X, L] U
$$

together with the Liouvillian (3), readily give Hamilton's equations

$$
\frac{d x}{d t}=\frac{\partial H}{\partial y}, \quad \frac{d y}{d t}=-\frac{\partial H}{\partial x} .
$$

There is however an important difference: the time translation operator $L$ is not the energy, and its spectrum may extend to $-\infty$. For example, if we have a harmonic oscillator with $H=\left(x^{2}+y^{2}\right) / 2$, the Liouvillian is

$$
L=y p_{x}-x p_{y}
$$

whose eigenvalues are all the integers, negative as well as positive. There is nothing wrong in that, since $L$ involves the unobservable shift operators $p_{x}=-i d / d x$ and $p_{y}=-i d / d y$, 
and therefore $L$ itself is not observable. Note that the solution of Hamilton's equations does not introduce non-observable components into the observable variables, because the Hamiltonian (contrary to the Liouvillian) involves only the observables $x$ and $y$.

It is easy to introduce into the above dynamical formalism a quantum system, with conjugate dynamical variables $q$ and $p$, as long as the two systems do not interact. In the Schrödinger picture, we have a wave function $\psi(x, y, q)$ whose evolution is given by

$$
\partial \psi / \partial t=K \psi
$$

where $K$ is "Koopmanian"

$$
K=L\left(x, y, p_{x}, p_{y}\right)+H(q, p) .
$$

In that wave function, the coordinates $x, y$, and $q$ may be entangled. In any case, we can obtain the reduced density matrices of the "classical" and quantum systems by means of partial traces on the other system, as usual.

The non-trivial problem is to introduce an interaction between the two systems. An apparent difficulty occurs in the Heisenberg picture because the equations of motion will mix variables of all kinds: classical observables, non-observables, and quantum operators. This still is acceptable, because we do not predict actual values for these variables, but only expectation values, $\langle A\rangle=\operatorname{tr}(\rho A)$, and no contradiction may occur. Anyway, we can always use the Schrödinger picture, where we know how to handle entangled wave functions.

The true difficulty, as we shall now see in a simple example, is that the correspondence principle fails. Consider two harmonic oscillators, with a bilinear coupling $k q x$, where $k$ is a constant. If we treat both of them classically, with a Hamiltonian

$$
H=\frac{1}{2}\left(q^{2}+p^{2}+x^{2}+y^{2}\right)+k q x,
$$

we obtain equations of motion

$$
\begin{array}{ll}
\dot{q}=p, & \dot{p}=-q-k x, \\
\dot{x}=y, & \dot{y}=-x-k q,
\end{array}
$$

and there are two characteristic frequencies, $\omega=\sqrt{1 \pm k}$, corresponding to the normal modes $(q \pm x)$. Exactly the same equations of motion appear in the Heisenberg picture for 
quantum mechanics. Likewise, in the Schrödinger picture, whenever there is a quadratic potential, the differential equation for the Wigner distribution is identical to the Liouville equation in classical mechanics [0]. In view of this formal agreement of classical and quantum mechanics, it is natural to demand that any hybridization of the system in Eq. (13) shall also give the same equations of motion. This is the definite benchmark we propose for an acceptable classical-quantum hybrid formalism. It is not obvious that this criterion can be achieved, because the hybrid system has no normal modes $(q \pm x)$.

Let us try to obtain the above equations of motion from a Koopmanian

$$
K=\frac{1}{2}\left(q^{2}+p^{2}\right)+\left(y p_{x}-x p_{y}\right)+K_{i}
$$

with a suitably chosen interaction term $K_{i}$. We cannot have both $\left[p, K_{i}\right]=-k x$ and $\left[y, K_{i}\right]=-k q$ together with $[y, p]=0$, because these equations are incompatible with Jacobi's identity

$$
\left[y,\left[p, K_{i}\right]\right]+\left[p,\left[K_{i}, y\right]\right]+\left[K_{i},[y, p]\right] \equiv 0
$$

The best result we were able to obtain was by "koopmanizing" the interaction term $k q x$ in the Hamiltonian (13). We have, from Eq. (3),

$$
K_{i}=i k q \partial / \partial y \equiv-k q p_{y}
$$

The resulting equations of motion are Eq. (15), unchanged, and

$$
\begin{aligned}
& \dot{q}=p, \quad \dot{p}=-q-k p_{y}, \\
& \dot{p}_{x}=p_{y}, \quad \dot{p}_{y}=-p_{x} .
\end{aligned}
$$

The last equation is necessary, because an unobservable variable $p_{y}$ appears in Eq. (19). The solution of (20) for $p_{y}$ is a superposition of $\sin t$ and $\cos t$ terms. When substituted into Eq. (19), these terms behave as a driving force with resonant frequency, so that $q$ and $p$ include terms behaving as $t \sin t$ and $t \cos t$. The amplitude of the quantum oscillator increases linearly with time, and energy is not conserved. It need not be: what is conserved is the Koopmanian, which includes a term $\left(y p_{x}-x p_{y}\right)$ whose spectrum extends to $-\infty$.

In conclusion, there is no mathematical inconsistency in the hybrid formalism that we proposed. However, it violates the correspondence principle which we would expect 
to hold exactly for a pair of oscillators with bilinear coupling. Therefore such a theory appears quite abnormal from the point of view of physics.

We thank Lajos Diósi for stimulating discussions. DRT was supported by a grant from

the Technion Graduate School. Work by AP was supported by the Gerard Swope Fund and the Fund for Encouragement of Research.

\section{References}

[1] C. Möller, in Les Théories Relativistes de la Gravitation, (Centre National de la Recherche Scientifique, Paris, 1962) pp. 15-29.

[2] N. Bohr, in Atti del Congresso Internazionale dei Fisici, Como (1927); reprinted in Nature 121, 78 and 580 (1928); N. Bohr, in New Theories in Physics (International Institute of Intellectual Cooperation, Paris, 1939) pp. 11-45.

[3] O. Hay and A. Peres, Phys. Rev. A 58, 116 (1998).

[4] O. V.Prezhdo and V. V. Kisil, Phys. Rev. A 56, 162 (1997); J. Caro and L. L. Salcedo, Phys. Rev. A 60, 842 (1999); L. Diósi, N. Gisin, and W. T. Strunz, Phys. Rev. A 61, 022108 (2000); and references therein.

[5] J. M. Jauch, Helv. Phys. Acta 37, 293 (1964).

[6] B. O. Koopman, Proc. Nat. Acad. Sci. 17, 315 (1931).

[7] A. Peres, Quantum Theory: Concepts and Methods (Kluwer, Dordrecht, 1995), pp. 312-319.

[8] E. Wigner, Phys. Rev. 40, 749 (1932).

[9] M. Reed and B. Simon, Methods of Modern Mathematical Physics, vol. 1, Functional Analysis (Academic Press, New York, 1972); vol. 2, Fourier Analysis, SelfAdjointness (Academic Press, New York, 1975).

[10] A. Peres and D. Terno, Phys. Rev. E 53, 284 (1996). 